\title{
Suppression of Allergic Reactions by Dehulled Adlay in Association with the Balance of Th1/Th2 Cell Responses
}

\author{
Hsin-Yi Hsu,${ }^{\dagger}$ Bi-Fong Lin,${ }^{\ddagger}$ Jin-Yuarn Lin,${ }^{\ddagger}$ Ching-Chuan KuO ${ }^{\dagger}$ AND \\ WENCHANG CHIANG $*, \dagger$
}

Graduate Institute of Food Science and Technology and Department of Agricultural Chemistry, National Taiwan University, Taipei, Taiwan, Republic of China

\begin{abstract}
Dehulled adlay is known as a natural Chinese medicine having antiallergic activity, although its mechanism remains unclear. This study examined the effects of dehulled adlay on antigen-specific antibody and cytokine production. Mice were immunized three times with ovalbumin (OVA) in alum adjuvant. It was found that oral administration of dehulled adlay in mice suppressed the production of IgE against OVA antigen. Serum anti-OVA $\lg _{2 a}$ antibody levels were significantly increased in mice after oral administration of dehulled adlay. Furthermore, the production of IL-2 by OVA-stimulated splenocytes was augmented in dehulled adlay-fed mice. Although dehulled adlay had no effect on the serum anti-OVA Ig $G_{1}$ antibody levels, it had a great capacity to reduce IL-5 secretion by means of OVA-stimulated splenocytes. Hydrothermal processes, including steaming and extrusion cooking, did not change the capacity of dehulled adlay to suppress lgE production. Three fractions of dehulled alday, including methanolic extract, warm water extract, and residue, were obtained. The methanolic extract exhibited the greatest capacity to reduce anti-OVA IgE production. These results suggest that dehulled adlay has a modulating ability to shift the balance from Th2 to Th1 dominance in the T cell mediated immune system and may be beneficial for the treatment of allergic disorders.
\end{abstract}

KEYWORDS: Dehulled adlay; antiallergic activity; IgE; Th1/Th2 cell responses

\section{INTRODUCTION}

Allergies are a common problem in developed countries. Coombs and Gell (I) classified hypersensitivity diseases into four types, including type I-IV reactions, on the basis of their mechanisms of injury to immunological tissue. The prevalence of allergic diseases varies with the types of allergy involved and the populations at risk. Atopic diseases, nasal (allergic rhinitis) and bronchial asthma, are typical type I hypersensitivity reactions. Type I reaction is mediated by the IgE antibody in humans, so the $\operatorname{IgE}$ antibody plays an important role in hypersensitivity diseases (2).

The existence of Th1/Th2 subsets in T-helper lymphocytes that differ in their cytokine secretion patterns and effector functions provides a framework for understanding normal and pathological immune responses (3). Allergic responses involving IgE-dependent mast cell degranulation and eosinophil accumulation in sites of inflammation are considered to be due to the development and activation of Th2 cells (4). This specific Th2 cell subset produces predominantly IL-4 and IL-5, which both play essential roles in antigen-induced eosinophil infiltration into inflammation sites. IL-4 is the major inducer of classswitching to IgE biosynthesis in B lymphocytes. Elevated serum

* Author to whom correspondence should be addressed (telephone 886 2-23660124; fax 886-2-23638673; e-mail chiang@ccms.ntu.edu.tw).

Graduate Institute of Food Science and Technology.

$\doteqdot$ Department of Agricultural Chemistry.
IgE levels induced by IL-4 are associated with eosinophil infiltration into the airway. In addition, IL-5, an eosinophil activating factor, induces eosinophil differentiation and prolongs their survival. In contrast, the Th1 cell subset mainly secretes cytokines such as IL-2 and IFN- $\gamma$, which inhibit eosinophil infiltration and $\operatorname{IgE}$ and $\mathrm{IgG}_{1}$ secretion and enhance $\operatorname{IgG}_{2 \mathrm{a}}$ secretion in vivo (5-8). Because evidence suggests that the Th1 and Th2 types of reactions are reciprocally regulated in vivo $(6,8,9)$, modulation of the Th1/Th2 balance, namely, shifting the balance from Th2 to Th1 dominance, should be a rational strategy for treating allergic diseases involving Th2 cells.

Various kinds of Chinese medicine have long been used to treat allergic disorders in Asian countries, but little is known about their effective mechanisms. Adlay (soft-shelled job's tears, Coix lachryma-jobi L. var. ma-yuen Stapf) is an annual crop belonging to the Gramineae family. The seed of adlay has long been used in China to treat warts, chapped skin, rheumatism, and neuralgia and as an anti-inflammatory and antihelmintic agent (10). In addition, adlay has stomachic, diuretic, antipholgistic, anodynic, antispasmodic, and antitumor effects, according to the folklore of China and Japan. Adlay is reported to have various immunomodulatory activities. Yamada et al. have purified acidic heteroglycans with an anticomplementary activity from adlay seed (11). Otsuka et al. found that benzoxazinoid compounds in the root of adlay showed considerable inhibitory effects toward histamine release from rat mast cells 
(12). Hidaka et al. demonstrated that adlay seeds could increase the activities of cytotoxic T-lymphocytes and natural killer cells (13). Seo and his colleagues reported that the methanolic extract of adlay seed has anti-inflammatory properties, which may, in part, involve the inhibition of nitric oxide and superoxide anion radical production by activated macrophages (14). Kuo et al. demonstrated that methanolic extracts of adlay suppressed TPAinduced superoxide generation on neutrophil-like leukocytes (15) and may have anti-inflammatroy potential. Previous results from our laboratory showed that oral administration of dehulled adlay in mice suppressed the production of IgE against OVA antigen (16).

In this study, to evaluate the ability of dehulled adlay to modulate the Th1/Th2 balance, we investigated its ex vivo effect on Th1/Th2 cytokine production by cultured splenocytes derived from OVA-immunized BALB/c mice. We also examined the effect of the two hydrothermal processes on the antiallergic responses of dehulled adlay. Furthermore, we fractioned the dehulled adlay into three parts, including methanolic extract, water extract, and residues, to identify which fractions of dehulled adlay contain the antiallergic effects.

\section{MATERIALS AND METHODS}

Source of Adlay. Adlay was purchased from a farmer who planted Taichung Shuenyu No. 4 (TCS4) of Coix lachryma-jobi L. var. ma-yuen Stapf in Taichung, Taiwan, in March of 1997 and harvested it in July of the same year. The air-dried adlay seeds were dehulled, blended into powder, and screened through a 20-mesh sieve (aperture $=0.94 \mathrm{~mm}$ ).

Processes and Products of Dehulled Adlay. The products of two hydrothermal processes (steaming and extrusion cooking) applied to dehulled adlay were prepared

Steamed Samples. Dehulled adlay seeds were soaked in cold water $(1: 1.5, \mathrm{w} / \mathrm{v})$ for $4 \mathrm{~h}$ and then steamed at $100{ }^{\circ} \mathrm{C}$ for $1 \mathrm{~h}$ in an electric rice cooker. After steaming, the steamed samples were freeze-dried in a freeze-drier (model SFD-25, Chang Juing Co., Kaohsiung, Taiwan), blended into powder using a mill (Chien Shin Co., Taipei, Taiwan) with 60 standard mesh, and then stored at $-20^{\circ} \mathrm{C}$ until use.

Extrusion Cooking Samples. Powdered dehulled adlay of extrusion was carried out on a pilot scale twin screw extruder (model BC-45, Clextral Co.). The extruder was fitted with co-rotating, intermeshing twin screws, and the barrel was heated using induction heating. The screw profile has a series of segments, including long feeding screws, short feeding screws, two high pressure building screws, and mixing screws, which reduced the pressure before the extrudate exited through the die. The extrusion conditions were as follows: screw speed, 120 $\mathrm{rpm}$; temperature profile in the barrel zones toward the die plate, room temperature, 60,100 , and $140{ }^{\circ} \mathrm{C}$; die diameter, $4 \mathrm{~mm}$; feed rate, 270 $\mathrm{g} / \mathrm{min}$; and feed moisture, $15 \%$ (wet basis).

Fractionation Procedure for Dehulled Adlay. The powder of dehulled adlay $(5 \mathrm{~kg}$ ) was extracted with $15 \mathrm{~L}$ of methanol, continuously, at room temperature for 2 weeks. The plant material was filtered off, and the methanolic extracts were combined and concentrated to dryness under reduced pressure by a rotatory vaccum evaporator (Eyela, Tokyo, Japan). After methanolic extraction, the plant material was continuously extracted with warm water at $50{ }^{\circ} \mathrm{C}$ for $30 \mathrm{~min}$. The plant material was filtered off to obtain warm water extract and extraction residue. These two fractions were dried using a freeze-dryer (model SFD-25, Chang Juing Co., Kaohsiung, Taiwan). All three fractions of dehulled adaly were stored at $-20^{\circ} \mathrm{C}$ until use.

Preparation of Animal Diets. Experiment 1. Diets were substituted with $0,10,20$, or $50 \%$ raw dehulled adlay on the basis of the AIN-76 formula (17). The chemical composition of the dehulled adlay was crude protein, $17.5 \%$; crude fat, $8.3 \%$; crude fiber, $2.6 \%$; and non-nitrogen free extract, $69.3 \%$ (18). These percentages were used to calculate the percentage of substitution of casein, soybean oil, cellulose, and cornstrarch.
Table 1. Composition of the Experimental Diets (Grams per Kilogram of Diet) ${ }^{a}$

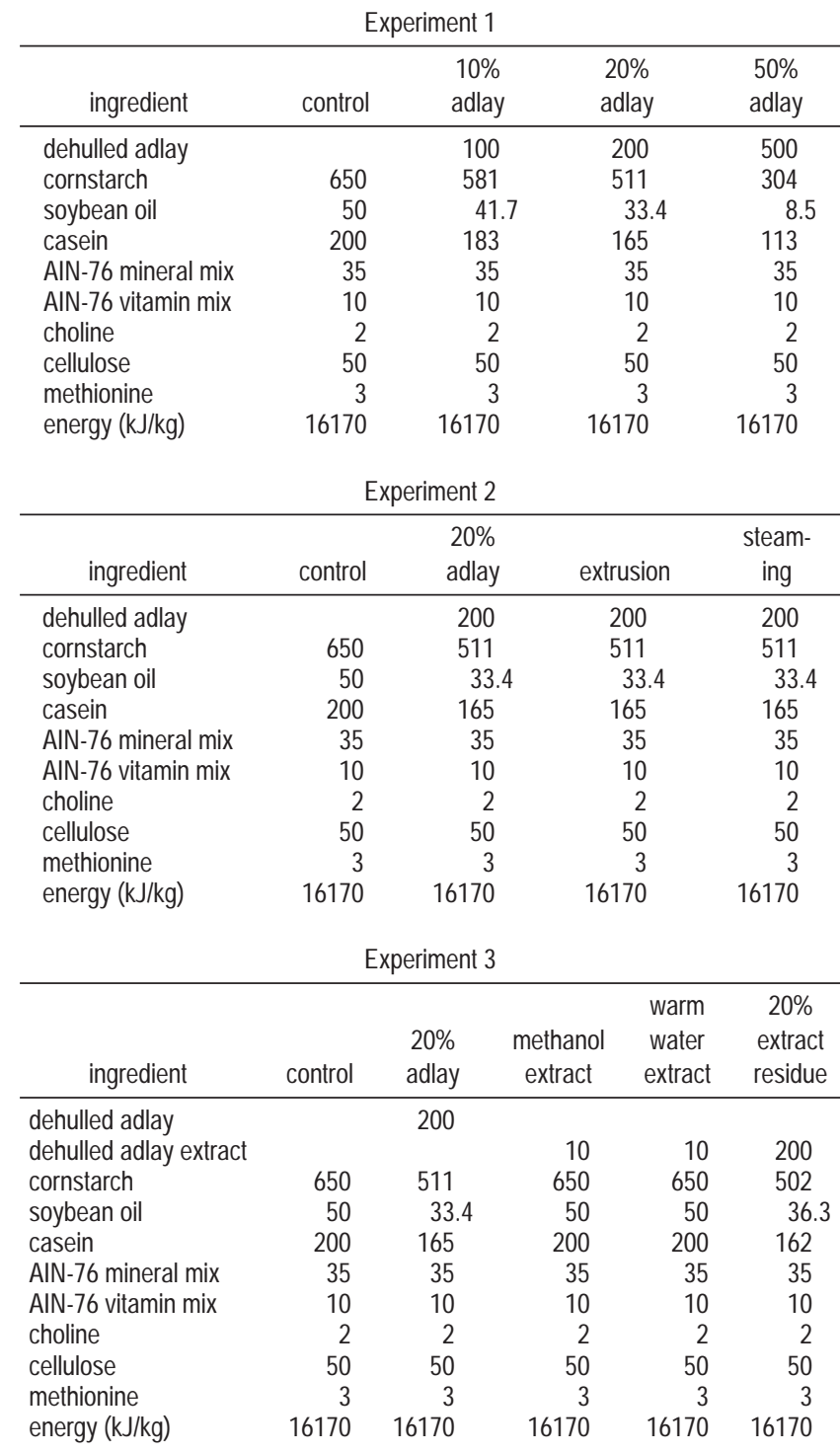

${ }^{a}$ Casein, methionine, and choline bitartrate, Sigma Chemical Co. (St. Louis, MO); soybean oil, President Co. (Tainan, Taiwan); cornstarch, Samyang Genex Co. (Seoul, Korea); cellulose, ARBOCEL, type BE 600/300, J. Rettenmaier and Söhne (Ellwangen-Holzmühle, Germany); AIN-76 mineral mixture and AIN-76 vitamin mixture, ICN Biochemicals, Inc. (Costa Mesa, CA).

Experiment 2. Diets were substituted with $20 \%$ raw, steamed, or extruded dehulled adlay on the basis of the AIN-76 formula.

Experiment 3. Diets were supplemented with $20 \%$ raw material, $1 \%$ methanolic extract, $1 \%$ warm water extract, or $20 \%$ extraction residue on the basis of the AIN-76 formula. Different components of the diet were added and mixed homogeneously. Soybean oil was added last. The diets were mixed again, meshed, and then sealed and stored at $-20{ }^{\circ} \mathrm{C}$. The compositions of the animal diets are shown in Table 1.

Animals and Experimental Design. Male BALB/c mice between 5 and 6 weeks of age were purchased from the Animal Centre of the College of Medicine at National Taiwan University (Taipei, Taiwan). The mice were kept in an air-conditioned, pathogen-free room at a temperature of $23 \pm 2{ }^{\circ} \mathrm{C}$ on a regulated 12-h light/dark cycle. They were housed individually in stainless steel wire cages and fed a nonpurified diet (Lab Rodent Chow 5001, Ralston Purina, St. Louis, MO) before being fed with the experimental diets. Animal care and handling conformed to the National Institutes of Health's Guide for the Care and Use of Laboratory Animals (National Research Council, 1985). Each mouse was fed an experimental diet starting from 8 weeks 
of age. The mice were given free access to the test diets throughout the experiment. The mice were weighed twice per week, and food consumption was measured every $2-3$ days. Each group included eight mice. Mice were immunized three times with ovalbumin (OVA) in alum adjuvant every 2 weeks. Briefly, after 6 weeks of consuming the test diets, the mice were first immunized by means of an intraperitoneal injection of $0.1 \mathrm{~mL}$ of PBS solution containing ovalbumin (OVA, 2 $\mu \mathrm{g})$ with aluminum hydroxide as the adjuvant. Then the mice were immunized 2 weeks later with $6 \mu \mathrm{g}$ of OVA plus the same adjuvant, and this procedure was repeated 2 weeks later (19). Blood was obtained on day 35 from the retro-orbital venous plexus and centrifuged at $12000 \mathrm{~g}$ for $10 \mathrm{~min}$. Serum was collected and stored at $-20{ }^{\circ} \mathrm{C}$ for future assays.

Determination of Serum Anti-ovalbumin Antibody Levels. Serum anti-OVA $\operatorname{IgE}, \operatorname{IgG}_{1}$, and $\mathrm{IgG}_{2 \mathrm{a}}$ antibody titers were measured by means of ELISA. Briefly, 96-well flat-bottom microtiter plates (Nunc 442404, Roskilde, Denmark) were coated with $10 \mu \mathrm{g} / \mathrm{mL}$ of OVA solution in $0.1 \mathrm{M} \mathrm{NaHCO}_{3}$ buffer, $\mathrm{pH}$ 8.2. After overnight incubation at $4{ }^{\circ} \mathrm{C}$, the plates were washed three times with PBS solution and blocked with $1 \% \mathrm{BSA}-\mathrm{PBS}$ for $2 \mathrm{~h}$ at room temperature. After they had been washed three times with PBST [PBS containing $0.05 \%(\mathrm{v} / \mathrm{v})$ Tween 20, washing buffer], $100 \mu \mathrm{L}$ of test sera diluted with BSA-PBS buffer was added to the wells, sera for $\operatorname{IgE}, \operatorname{IgG}_{1}$, and $\operatorname{IgG}_{2 \mathrm{a}}$ were diluted 1:10, 1:1000, and 1:100, respectively, and incubated overnight at $4{ }^{\circ} \mathrm{C}$ (IgE) or for $2 \mathrm{~h}$ at room temperature $\left(\operatorname{IgG}_{1}\right.$ and $\left.\operatorname{IgG}_{2 \mathrm{a}}\right)$. Plates were then washed with PBS-Tween 20 three times, and either biotin-conjugated antimouse $\mathrm{IgE}, \mathrm{IgG}_{1}$, or $\mathrm{IgG}_{2 \mathrm{a}}$ (Pharmingen, Becton Dickinson Co. Fullerton, CA) diluted in BSA-PBS buffer was added, followed by $1 \mathrm{~h}$ of incubation at room temperature. After washing, $0.1 \mathrm{~mL}$ of avidin-horseradish peroxidase (ImmunoPure, Pierce, Rockford, IL) for $\mathrm{IgG}_{1}$ and $\mathrm{IgG}_{2 \mathrm{a}}$ and $0.1 \mathrm{~mL}$ of avidin-conjugated alkaline phosphatase (Sigma Chemical Co., St. Louis, MO) for IgE were added to the wells. After $1 \mathrm{~h}\left(\mathrm{IgG}_{1}\right.$ and $\mathrm{IgG}_{2 \mathrm{a}}$ ) or $2 \mathrm{~h}$ (IgE) of incubation, the plates were washed five times, and $0.1 \mathrm{~mL}$ of ABTS [5.5 mg of 2,2 azinobis(3-ethylbenz)thiazoline6-sulfonic acid (Sigma Chemical Co.)] in $10 \mathrm{~mL}$ of citrate buffer containing $0.03 \% \mathrm{H}_{2} \mathrm{O}_{2}$ for $\operatorname{IgG}_{1}$ and $\mathrm{IgG}_{2 \mathrm{a}}$ and $0.1 \mathrm{~mL}$ of $p$-nitrophenyl phosphate aqueous solution (Sigma Chemical Co.) for IgE were added to each well and left in the dark for $\sim 30 \mathrm{~min}$ at room temperature. Absorbencies were measured at $405 \mathrm{~nm}$ for IgE and at $415 \mathrm{~nm}$ for $\mathrm{IgG}$, respectively, with an OPTImax tunable microplate reader (Molecular Devices, Sunnyvale, CA). The results were expressed in ELISA units (EU): $\mathrm{EU}=\left(A_{\text {sample }}-A_{\text {blank }}\right) /\left(A_{\text {positive }}-A_{\text {blank }}\right)$.

Cytokine Assay. Levels of cytokines produced by spleen cells were measured as previously described $(20)$. The spleen cells $\left(5 \times 10^{6}\right.$ cells/ $\mathrm{mL}$ ) from immunized mice were isolated and suspended in 10\% FBS/ RPMI 1640 culture medium and then cultured in the absence or presence of OVA $(20 \mu \mathrm{g} / \mathrm{mL})$ for $48 \mathrm{~h}$ at $37{ }^{\circ} \mathrm{C}$ in the $\mathrm{CO}_{2}$ incubator. After incubation, supernatants were collected and stored at $-80{ }^{\circ} \mathrm{C}$ until analyzed. The cytokines were measured by means of sandwich-ELISA. Briefly, 96-well flat-bottom microtiter plates were coated with anticytokine antibody diluted in $\mathrm{NaHCO}_{3}$ buffer, $\mathrm{pH}$ 9.6. After overnight incubation at $4{ }^{\circ} \mathrm{C}$, the plates were washed three times and blocked with BSA $(30 \mathrm{~g} / \mathrm{L})$ at $37^{\circ} \mathrm{C}$. After three washings with PBS containing $0.5 \mathrm{~mL}$ of Tween $20 / \mathrm{L}, 0.1 \mathrm{~mL}$ of sample was added for $2 \mathrm{~h}$ at $37^{\circ} \mathrm{C}$. The plates were then washed with PBS buffer containing $0.5 \mathrm{~mL}$ of Tween 20/L, and biotin-conjugated anti-cytokine antibody diluted in buffer $\left(10 \mathrm{~g}\right.$ of BSA/L of PBS) was added and incubated at $37^{\circ} \mathrm{C}$ for $1 \mathrm{~h}$. After washing, streptavidin-conjugated peroxidase was added for an additional hour. After the wells had been washed, $0.1 \mathrm{~mL}$ of enzyme substrate (ABTS) was added to each well, and the plate was left in a dark room for $\sim 30 \mathrm{~min}$. The plates were read in a microplate autoreader at $415 \mathrm{~nm}$. The sensitivity of sandwich-ELISA used in our experiment was $8 \mathrm{pg} / \mathrm{mL}$ for IL- 4 and IL-5 and 4 units $/ \mathrm{mL}$ for IL-2.

Statistical Analysis. The results were expressed as mean \pm SD. Statistical analysis of the difference between the results of the control and treated experimental groups was carried out using Student's $t$ test. $p$ values below 0.05 were considered to be significant.

\section{RESULTS}

Whole Body Growth and Feed Intake. Animals on the adlay diet appeared to be healthy, showing no pathological signs

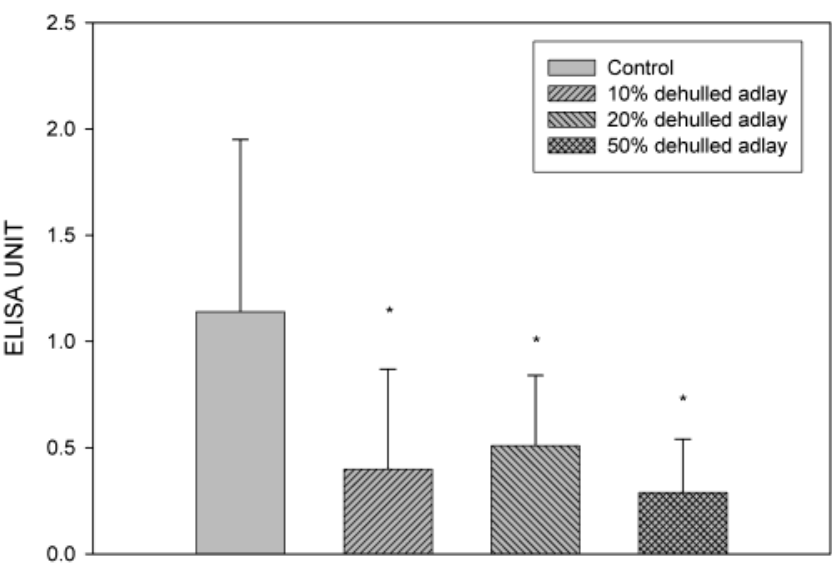

Figure 1. Effects of different dosages of dehulled adlay on OVA-specific $\lg E$ levels in serum. Mice consumed the test diets for 6 weeks and then were intraperitoneally immunized with OVA plus alum. After immunization and every day thereafter, the mice were continuously administered the test diets. Sera were obtained at day 35 from the retro-orbital venous plexus after the first immunization. The results are expressed as the mean $\pm S D$ of eight BALB/c mice per group. Mice that consumed dehulled alday-containing diets exhibited significant effects compared with the control mice, as evaluated using Student's $t$ test $\left({ }^{*}, p<0.05\right)$.

or abnormalities during the feeding period. There was no significant difference among any of the treatments for body weight gain of the mice. The feed intake (grams per day per mouse) was the same in all of the groups tested. The rate of growth was also similar in all of the groups tested (data not shown).

Effect of Different Dosages of Dehulled Adlay on Serum Anti-ovalbumin Antibody Levels. To determine whether antigen-specific IgE production was suppressed in OVAimmunized mice by various doses of diets containing dehulled adlay, we obtained serum from OVA-immunized mice 35 days after immunization. In OVA-immunized mice, antigen-specific $\mathrm{IgE}$ production was significantly increased at the time point examined after immunization as compared with mice without immunization (anti-OVA IgE levels of OVA-immunized mice and mice without immunization were $1.14 \pm 0.81$ and $0.05 \pm$ $0.02 \mathrm{EU}$, respectively). Antigen-specific IgE production decreased significantly in OVA-immunized mice treated with diets containing 10,20 , and $50 \%$ dehulled adlay as compared with mice fed with the control diet $(p<0.05)$ (Figure 1). Furthermore, the anti-OVA $\operatorname{IgG}_{2 \mathrm{a}}$ antibody levels of the mice fed diets containing 20 and $50 \%$ dehulled adlay seemed to be higher than that of the mice fed the control diet $(p<0.05)$ (Table 2). However, the anti-OVA $\operatorname{IgG}_{1}$ antibody levels showed no significant differences among all of the experimental groups (Table 2).

Regulatory Effect of Dehulled Adlay on the Balance of Th1/Th2 Cell Responses in OVA-Immunized Mice. To determine the regulatory effect of dehulled adlay on Th1/Th2 cell responses in OVA-immunized mice, dehulled adlay was orally administered every day from day 42 before immunization. IL-2 (Th1 cytokine) and IL4 and IL-5 production (Th2 cytokines) in splenocytes stimulated with or without OVA was assayed. The production of IL-2 by OVA-stimulated splenocytes increased in all of the dehulled adlay-fed mice $(p<0.05)$ (Table 3). However, feeding dehulled adlay had no different effect on IL-4 levels (Table 3), but it greatly reduced IL-5 secretion by both medium alone and OVA-stimulated splenocytes (Table 3). These results indicated that the dehulled adlay changed the 
Table 2. Effects of Different Dosages of Dehulled Alday on Antigen-Specific $\lg _{1}$ and $\lg _{2 a}$ Levels in Seruma

\begin{tabular}{lcc}
\hline \multicolumn{1}{c}{ group } & $\begin{array}{c}\text { anti-OVA IgG } \\
\text { (ELISA unit) }\end{array}$ & $\begin{array}{c}\text { anti-OVA IgG } \\
\text { (ELISA unit) }\end{array}$ \\
\hline control & $3.95 \pm 1.47$ & $1.75 \pm 1.56$ \\
$10 \%$ dehulled adlay & $4.29 \pm 1.49$ & $1.88 \pm 0.92$ \\
$20 \%$ dehulled adlay & $4.73 \pm 0.84$ & $4.83 \pm 4.14^{*}$ \\
$50 \%$ dehulled adlay & $5.08 \pm 1.02$ & $3.14 \pm 1.78^{*}$
\end{tabular}

\begin{abstract}
${ }^{a}$ Mice consumed the test diets for 6 weeks and then were intraperitoneally immunized with OVA plus alum. After immunization and every day thereafter, the mice were continuously administered the test diets. Sera were obtained at day 35 from the retro-orbital venous plexus after the first immunization. The results are expressed as the mean $\pm S D$ of eight BALB/c mice per group. Mice that consumed dehulled alday-containing diets exhibited significant effects compared with the control mice, as evaluated using Student's $t$ test $\left({ }^{*}, p<0.05\right)$.
\end{abstract}

balance of Th1/Th2 cell immune responses from Th2-dominant to Th1-dominant in OVA-immunized mice.

Effect of Hydrothermal Processing Products of Dehulled Adlay on Anti-OVA IgE Level. Can hydrothermal processing procedures reduce the antiallergic activity of dehulled adlay? In this study, we investigated the effects of two products made from dehulled adlay using two kinds of hydrothermal processes (namely, steamed and extruded products) on their antiallergic capacity. The results showed that extrusion cooking and steaming of dehulled adlay did not change its capacity to suppress IgE production (Figure 2).

Evaluation of the Major Antiallergic Fraction from Dehulled Adlay. What is the major active fraction of dehulled adlay having antiallergic activity? We also conducted crude fractionation in this study. Dehulled adlay was extracted with methanol and hot water successively. Three fractions of dehulled alday, including methanolic extract, warm water extract, and extraction residues, were obtained. Figure 3 shows that the methanolic extract exhibited the greatest capacity to reduce antiOVA IgE production. The anti-OVA $\mathrm{IgG}_{1}$ antibody levels were not significantly different among the experimental groups (Table 4). Furthermore, the mice fed diets containing $1 \%$ of dehulled adlay methanolic extracts had much higher anti-OVA $\operatorname{IgG}_{2 \mathrm{a}}$ antibody levels $(p<0.05)$ (Table 4).

\section{DISCUSSION}

There has been a considerable increase in the incidence of atopic diseases in industrialized societies during the past 30 years, and it has been estimated that at least $20 \%$ of the population worldwide is susceptible to atopic disease (21). The number of patients that suffer form type I allergic diseases, such as allergic rhinitis and brochial asthma, has been increasing year by year in Taiwan $(22,23)$. Commercial drugs might work to alleviate the patient's symptoms but do not affect the pathogenesis. The components of traditional Chinese medicines have been intensively studied for treating allergic diseases in recent years.

Chinese herbal medicines, including Bu-zhong-yi-qi-tang (24), Perilla frutescens Britton (25), and royal jelly (26), have been reported to suppress allergic reactions. These agents improve the Th cell responses in the host, causing a switch from a Th2-dominant to a Th1-dominant pattern, and suppress IL-4 production by Th2 cells, thereby alleviating allergies.

It has been found that dietary ingredients play a role in antiallergic and anti-inflammatory effects. Dietary oxidized oil influences the levels of type 2 T-helper cell-related antibody and inflammatory mediators (20). Dietary oligosaccharide raffinose suppresses serum immunoglobulin E response through suppression of Th2-type immune response against oral antigen in the lymphoid organs located in or near the intestine (27). The flavonoid compound hesperidin is an effective component of Citrus fruit with antiallergic action against the type I reaction (28).

Adlay has long been used in Chinese medicine to treat inflammatory disease in China and Japan. In our previous research, adlay was shown to have the potential capacity to reduce OVA-specific IgE biosynthesis (16). The present data showed that oral administration of dehulled adlay significantly decreased the serum level of antigen-specific IgE in OVAimmunized mice (Figure 1) and increased the serum level of antigen-specific $\mathrm{IgG}_{2 \mathrm{a}}$, although the antigen-specific $\mathrm{IgG}_{1}$ responses were not consistently lowered (Table 2). Nevertheless, $\mathrm{IgE}$ is one of the major effectors in allergic response; through cross-linking with allergens it begins the pathophysiological cascade of the allergy reaction (4). Therefore, the possible mechanism of the suppression in IgE production by dehulled adlay is worthy of further investigation.

Helper T cells are divided into two subtypes, Th1 and Th2, that produce different cytokines and therefore perform distinct effector functions (4). The existence of these effector subtypes provides a model for understanding the pathogenesis of pathophysiologic conditions such as allergic diseases and the $\operatorname{IgE}$ response to allergens. Th1 and Th2 cells derive from the same precursors in response to different stimuli and are distinguished most clearly by the cytokines they produce. Th1 secrete interleukin (IL)-2, interferon (IFN)- $\gamma$, tumor necrosis factor (TNF)- $\alpha$, lymphotoxin (LT), and other cytokines that activate macrophages and are responsible for cell-mediated immunity against intracellular microbes but antagonize the $\operatorname{IgE}$ response (4). In contrast, Th2 cells produce IL-4, IL-5, IL-9, and IL-13, which are responsible for the antibody response, including $\operatorname{IgE}$ production, and inhibit several macrophage functions. The principal effector functions of Th2 cells are IgE production and IgE-dependent eosinophil and mast cell-mediated immune reactions (29).

Allergen-induced Th1 and Th2 cytokine imbalance are important cause of inflammation in allergic response. The present results showed that the production of IL-2 by OVAstimulated splenocytes was increased in dehulled adlay-fed mice. Although dehulled adlay had no significant effect on IL-4 levels, it had a great capacity to reduce IL-5 secretion by OVAstimulated splenocytes (Table 3). IL-5, which is a lineagespecific eosinophil growth factor, increases the formation of eosinophils from progenitor cells and, in concert with CCR3 active chemokines, increases their trafficking to sites of allergic inflammation (30-32). In subjects with asthma, allergendriven IL-5 production correlated with bronchial hyperreactivity (33). The present study showed that dehulled adlay suppressed the acceleration of antigen-specific IgE production in OVAimmunized mice by restoring of Th cell responses from a Th2dominant to a Th1-dominant pattern. This result suggests that dehulled adlay, which shows immunomodulatory activity, may suppress allergic reactions.

In addition, chemical changes during extrusion cooking have been reported in several studies (34-36). Little is known about the effects of extrusion parameters on phytochemical bioavailability and stability (34-36). Therefore, the effects of hydrothermal processes on the antiallergic properties of dehulled adlay have also been evaluated here. Steaming, a traditional cooking process, is a high-temperature-long time process. However, extrusion cooking is a high-temperature-high shearing-short 
Table 3. Effects of Dietary Adlay on Cytokine Responses by OVA-Sensitized Mice Splenocytes ${ }^{a}$

\begin{tabular}{|c|c|c|c|c|c|c|}
\hline \multirow[b]{2}{*}{ group } & \multicolumn{2}{|c|}{ IL-2 (units/106 cells) } & \multicolumn{2}{|c|}{ IL-4 (pg/106 cells) } & \multicolumn{2}{|c|}{ IL-5 (units/106 cells) } \\
\hline & medium alone & OVA & medium alone & OVA & medium alone & OVA \\
\hline control & $1.33 \pm 0.09$ & $2.51 \pm 0.22$ & $5.37 \pm 0.56$ & $5.48 \pm 0.48$ & $68.1 \pm 2.8$ & $86.5 \pm 18.5$ \\
\hline $10 \%$ adlay & $1.41 \pm 0.10$ & $4.26 \pm 0.64^{*}$ & $5.66 \pm 0.87$ & $6.53 \pm 1.21$ & $53.2 \pm 13.0^{*}$ & $78.0 \pm 19.9$ \\
\hline $20 \%$ adlay & $2.44 \pm 0.20^{*}$ & $3.52 \pm 0.39^{*}$ & $6.37 \pm 0.88$ & $6.41 \pm 1.50$ & $53.17 \pm 8.3^{\star}$ & $66.0 \pm 27.8^{*}$ \\
\hline $50 \%$ adlay & $2.39 \pm 0.16^{*}$ & $3.52 \pm 0.50^{*}$ & $6.76 \pm 0.76$ & $6.25 \pm 1.00$ & $42.1 \pm 4.5^{\star}$ & $55.8 \pm 11.7^{*}$ \\
\hline
\end{tabular}

${ }^{a}$ Effects of different dosages of dehulled adlay on cytokine production from splenocyte culture. Splenocytes, at a concentration of $5 \times 10^{6}$ cells $/ \mathrm{mL}$, were cultured with the medium described earlier in the absence or presence of OVA $(20 \mu \mathrm{g} / \mathrm{mL})$ for $48 \mathrm{~h}$. Cytokine secretions by single-cell suspensions of splenocytes were measured by means of sandwich-ELISA. The results are expressed as the mean \pm SD of eight BALB/c mice per group. Mice that consumed dehulled alday-containing diets exhibited significant effects compared with the control mice, as evaluated using Student's $t$ test $\left({ }^{*}, p<0.05\right)$.

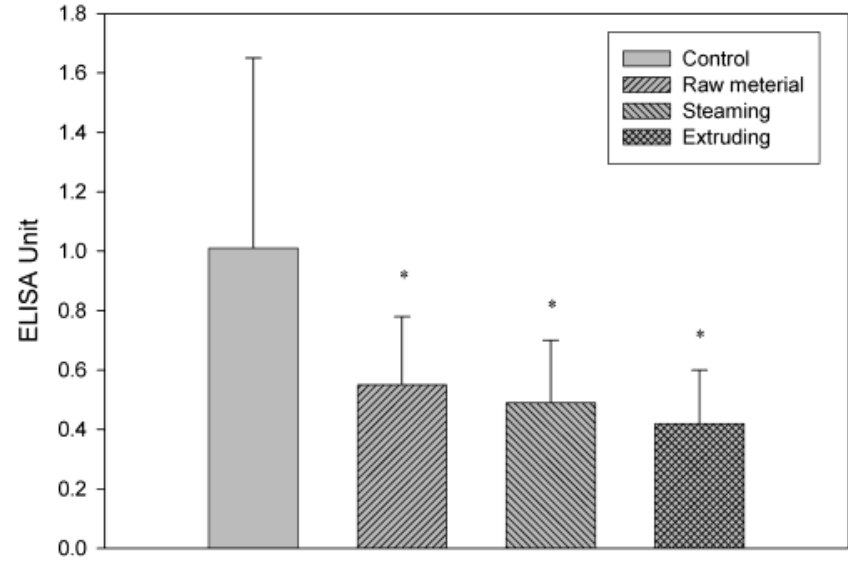

Figure 2. Effects of different processing products of dehulled adlay on OVA-specific lgE levels in serum. Mice were fed the test diets for 6 weeks and then were intraperitoneally immunized with OVA plus alum. After immunization and every day thereafter, the mice were continuously administered the test diets. Sera were obtained at day 35 from the retroorbital venous plexus after immunization. The results are expressed as the mean $\pm S D$ of eight BALB/c mice per group. Mice that consumed dehulled alday-containing diets exhibited significant effects compared with control mice, as evaluated using Student's $t$ test $\left(^{*}, p<0.05\right)$.

time process. The results show that the antiallergic activity of adlay was preserved in the steamed or extruded products (Figure 2). We have shown that the components having antiallergic activities in dehulled alday remained stable during hydrothermal processing, indicating that the cooking process has no effect on the functional components of the dehulled adlay.

There are many biologically active phytochemicals in adlay, and some of them have been isolated and identified $(12,37-$ 41). Otsuka et al. found that benzoxazinoid compounds, such as coixol, exist in the root of adlay and show considerable inhibitory activity toward histamine release from rat mast cells (12). There are other biologically active substances, such as coixenolide, coixinic acid, coniferyl alcohol, syringic acid, ferulic acid, syringaresinol, 4-ketopinoresinol, mayuenolide, and others in adlay $(12,37-41)$. The phenolic compounds in methanolic extracts of adlay hulls (37), such as coniferyl alcohol, p-coumaric acid, syringic acid, ferulic acid, syringaresinol, 4-ketopinoresinol, and mayuenolide, have been shown to be antioxidants and anti-inflammatory agents $(37,42-48)$. In addition, we found that dehulled adlay contains various types of sterolic components (data not shown). Plant sterols and sterolins have been shown to exhibit anti-inflammatory, antineoplastic, and immune-modulating activity (49-52). In this study we found that the methanolic extract of dehulled adlay is the major active fraction suppressing OVA-specific IgE and elevating $\mathrm{IgG}_{2 \mathrm{a}}$ biosynthesis. Antiallergic constituents in the mechanolic fraction of dehulled adlay may be related to phenolic

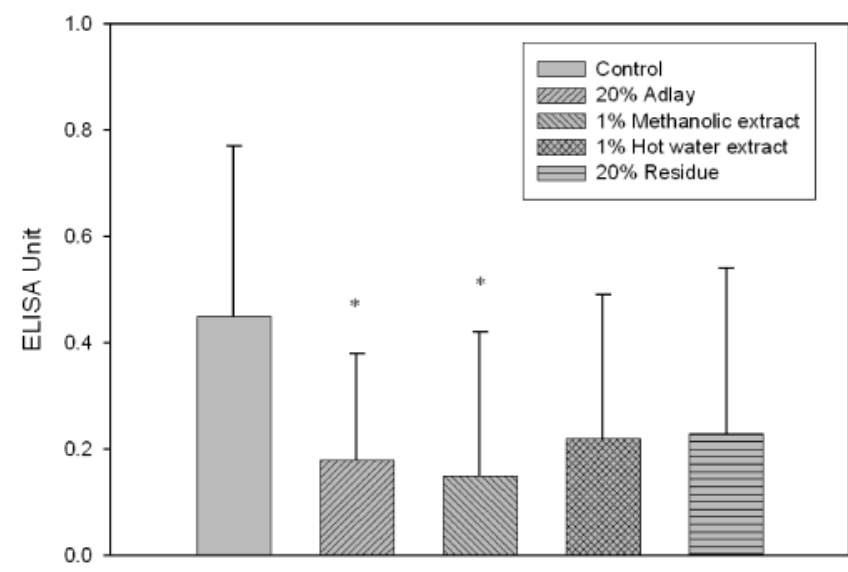

Figure 3. Effects of the subfracitons of dehulled adlay on OVA-specific $\lg$ E levels in serum. Mice were fed the test diets for 6 weeks and then were intraperitoneally immunized with OVA plus alum. After immunization and every day thereafter, the mice were continuously administered the test diets. Sera were obtained at day 35 from the retro-orbital venous plexus after immunization. The results are expressed as the mean \pm SD of eight BALB/c mice per group. Mice that consumed dehulled aldaycontaining diets exhibited significant effects compared with the control mice, as evaluated using Student's $t$ test $\left({ }^{*}, p<0.05\right)$.

Table 4. Effects of the Subfractions of Dehulled Alday on Antigen-Specific $\lg _{1}$ and $\lg _{2 a}$ Levels in Serum ${ }^{a}$

\begin{tabular}{lcc}
\hline \multicolumn{1}{c}{ group } & $\begin{array}{c}\text { anti-OVA IgG } \\
\text { (ELISA unit) }\end{array}$ & $\begin{array}{c}\text { anti-OVA IgG } \\
\text { (ELISA unit) }\end{array}$ \\
\hline control & $1.19 \pm 0.24$ & $0.41 \pm 0.17$ \\
raw material & $1.04 \pm 0.30$ & $0.59 \pm 0.49^{*}$ \\
methanolic extract & $1.13 \pm 0.18$ & $1.08 \pm 0.81^{*}$ \\
hot water extract & $0.98 \pm 0.39$ & $0.55 \pm 0.34$ \\
residue & $1.39 \pm 0.26$ & $0.36 \pm 0.22$
\end{tabular}

\footnotetext{
${ }^{a}$ Mice were fed the test diets for 6 weeks and then were intraperitoneally immunized with OVA plus alum. After immunization and every day thereafter, the mice were continuously administered the test diets. Sera were obtained at day 35 from the retro-orbital venous plexus after immunization. The results are expressed as the mean $\pm S D$ of eight $B A L B / c$ mice per group. Mice that consumed dehulled alday-containing diets exhibited significant effects compared with the control mice, as evaluated using Student's $t$ test $\left(^{*}, p<0.05\right)$.
}

or sterolic constituents. Whether some of these compounds can suppress allergic pathology development remains to be investigated.

This study demonstrated that oral treatment with dehulled adlay modulated the pattern of Th1/Th2 cytokine production, thus suppressing IgE biosynthesis in OVA-sensitized BALB/c mice. Our study suggested that the antiallergic properties of dehulled adlay may be beneficial in alleviating allergic symptoms. The antiallergic components of dehulled adlay are valuable and merit further investigation. 


\section{LITERATURE CITED}

(1) Coombs, R. R. A.; Gell, P. G. H.; Lachmann, P. J. Classification of allergic reactions responsible for clinical hypersensitivity and disease. In Clinical Aspects of Immunology; Gell, P. G. H., Coombs, R. R. A., Eds.; Blackwell Scientific Publications: Oxford, U.K., 1975.

(2) Serafin, W. E.; Austen, K. F. Mediators of immediate hypersensitivity reactions. N. Engl. J. Med. 1987, 317, 30-34.

(3) Mosmann, T. R.; Coffman, R. L. TH1 and TH2 cells: different patterns of lymphokine secretion lead to different functional properties. Аnпи. Rev. Immunol. 1989, 7, 145-173.

(4) Abbas, A. K.; Murphy, K. M.; Sher, A. Functional diversity of helper T lymphocytes. Nature 1996, 383, 787-793.

(5) Bellanti, J. A. Cytokines and allergic diseases: clinical aspects. Allergy Asthma Proc. 1998, 19, 337-341.

(6) Chin, J. E.; Hatfield, C. A.; Winterrowd, G. E.; Krzesicki, R. F.; Shull, K. L.; Fidler, S. F.; Kolbasa, K. P.; Brashler, J. R.; Griffin, R. L.; Fleming, W. E.; Justen, J. M.; Banitt, L. S.; Bundy, G. L.; Richards, I. M. Preclinical evaluation of anti-inflammatory activities of the novel pyrrolopyrimidine PNU-142731A, a potential treatment for asthma. J. Pharmacol. Exp. Ther. 1999, 290, 188-195.

(7) Mosmann, T. R.; Cherwinski, H.; Bond, M. W.; Giedlin, M. A.; Coffiman, R. L. Two types of murine helper T cell clone. I. Definition according to profiles of lymphokine activities and secreted proteins. J. Immunol. 1986, 136, 2348-2357.

(8) Viola, J. P.; Rao, A. Molecular regulation of cytokine gene expression during the immune response. J. Clin. Immunol. 1999, 19, 98-108

(9) Kapsenberg, M. L.; Wierenga, E. A.; Bos, J. D.; Jansen, H. M. Functional subsets of allergen-reactive human CD4+ T cells. Immunol. Today 1991, 12, 392-395.

(10) Li, S. C. Pen-t'sao kang mu (Systematic Pharmacopoeia); China, 1596.

(11) Yamada, H.; Yanahira, S.; Kiyohara, H.; Cyong, J.-C.; Otsuka, Y. Characterization of anti-complementary acidic heteroglycans from the seed of Coix lacryma-jobi var. ma-yuen. Phytochemistry 1987, 26, 3269-3275.

(12) Otsuka, H.; Hirai, Y.; Nagao, T.; Yamasaki, K. Anti-inflammatory activity of benzoxazinoids from roots of Coix lachrymajobi var. ma-yuen. J. Nat. Prod. 1988, 51, 74-79.

(13) Hidaka, Y.; Kaneda, T.; Amino, N.; Miyai, K. Chinese medicine, Coix seeds increase peripheral cytotoxic $\mathrm{T}$ and $\mathrm{NK}$ cells. Biotherapy 1992, 5, 201-203.

(14) Seo, W. G.; Pae, H. O.; Chai, K. Y.; Yun, Y. G.; Kwon, T. H.; Chung, H. T. Inhibitory effects of methanol extract of seeds of Job's tears (Coix lachryma-jobi L. var. ma-yuen) on nitric oxide and superoxide production in RAW 264.7 macrophages. Immunopharmacol. Immunotoxicol. 2000, 22, 545-554.

(15) Kuo, C. C.; Shih, M. C.; Kuo, Y. H.; Chiang, W. Antagonism of free-radical-induced damage of adlay seed and its antiproliferative effect in human histolytic lymphoma U937 monocytic cells. J. Agric. Food Chem. 2001, 49, 1564-1570.

(16) Shyu, M. L.; Lin, B. F.; Chiang, W. Effect of dehulled adlay on allergic responses of sensitized mice. Nutr. Sci. J. 1998, 23, $161-170$.

(17) Report of the American Institute of Nurtition ad hoc Committee on Standards for Nutritional Studies. J. Nutr. 1977, 107, 13401348.

(18) Hung, S. L.; Chen, Y. F.; Chiang, W. Amino acids, fatty acids and proximate composition of the seed of adlay. Food Sci. 1994, 21, 67-74.

(19) Chuang, Y. H.; Chiang, B. L.; Chou, C. C.; Hsieh, K. H. Different kinds of antigen-presenting cells exert different effects on T-helper cells development. Int. Arch. Allergy Immunol. 1996, 111, 366-371.

(20) Lin, B. F.; Lai, C. C.; Lin, K. W.; Chiang, B. L. Dietary oxidized oil influences the levels of type $2 \mathrm{~T}$-helper cell-related antibody and inflammatory mediators in mice. Br. J. Nutr. 2000, 84, 911-917.
(21) Romagnani, S. Atopic allergy and other hypersensitivities interactions between genetic susceptibility, innocuous and/or microbial antigens and the immune system. Curr. Opin. Immunol. 1997, 9, 773-775.

(22) Chiang, B. L. Molecular mechanisms of allergen-specific immunotherapy for atopic diseases. Zhonghua Min Guo Xiao Er Ke Yi Хие Hиi Za Zhi 1998, 39, 293-296.

(23) Lo, S. F.; Chiang, B. L.; Hsieh, K. H. Analysis of total IgE and allergen-specific IgE antibody levels of allergic children in Taiwan. Zhonghua Min Guo Xiao Er Ke Yi Xue Hui Za Zhi 1997, $38,375-380$.

(24) Kaneko, M.; Kishihara, K.; Kawakita, T.; Nakamura, T.; Takimoto, H.; Nomoto, K. Suppression of IgE production in mice treated with a traditional Chinese medicine, bu-zhong-yi-qi-tang (Japanese name: hochu-ekki-to). Immunopharmacology 1997, $36,79-85$.

(25) Makino, T.; Furuta, A.; Fujii, H.; Nakagawa, T.; Wakushima, H.; Saito, K.; Kano, Y. Effect of oral treatment of Perilla frutescens and its constituents on type-I allergy in mice. Biol. Pharm. Bull. 2001, 24, 1206-1209.

(26) Oka, H.; Emori, Y.; Kobayashi, N.; Hayashi, Y.; Nomoto, K. Suppression of allergic reactions by royal jelly in association with the restoration of macrophage function and the improvement of Th1/Th2 cell responses. Int. Immunopharmacol. 2001, 1, 521532.

(27) Nagura, T.; Hachimura, S.; Hashiguchi, M.; Ueda, Y.; Kanno, T.; Kikuchi, H.; Sayama, K.; Kaminogawa, S. Suppressive effect of dietary raffinose on T-helper 2 cell-mediated immunity. $\mathrm{Br}$. J. Nutr. 2002, 88, 421-427.

(28) Matsuda, H.; Yano, M.; Kubo, M.; Iinuma, M.; Oyama, M.; Mizuno, M. [Pharmacological study on citrus fruits. II. Antiallergic effect of fruit of Citrus unshiu Markovich (2). On flavonoid components]. Yakugaku Zasshi 1991, 111, 193-198.

(29) Robinson, D. S. Th-2 cytokines in allergic disease. Br. Med. Bull. 2000, 56, 956-968.

(30) Broide, D. H. Molecular and cellular mechanisms of allergic disease. J. Allergy Clin. Immunol. 2001, 108, S65-S71.

(31) Cho, S. H.; Stanciu, L. A.; Begishivili, T.; Bates, P. J.; Holgate, S. T.; Johnston, S. L. Peripheral blood CD4+ and CD8+ T cell type 1 and type 2 cytokine production in atopic asthmatic and normal subjects. Clin. Exp. Allergy 2002, 32, 427-433.

(32) Gleich, G. J. Mechanisms of eosinophil-associated inflammation. J. Allergy Clin. Immunol. 2000, 105, 651-663.

(33) Till, S.; Dickason, R.; Huston, D.; Humbert, M.; Robinson, D.; Larche, M.; Durham, S.; Kay, A. B.; Corrigan, C. IL-5 secretion by allergen-stimulated $\mathrm{CD} 4+\mathrm{T}$ cells in primary culture: relationship to expression of allergic disease. J. Allergy Clin. Immunol. 1997, 99, 563-569.

(34) Bangoula, D.; Parent, J. P.; Vellas, F. Nutritive value of white lupin (Lupinus albus var. Lutop) in rainbow trout (Oncorhynchus mykiss). Effect of extrusion cooking. Reprod. Nutr. Dev. 1993, $33,325-334$

(35) Camire, M. E. Chemical changes during extrusion cooking. Recent advances. Adv. Exp. Med. Biol. 1998, 434, 109-121.

(36) Omueti, O.; Morton, I. D. Development by extrusion of soyabari snack sticks: a nutritionally improved soya-maize product based on the Nigerian snack (kokoro). Int. J. Food Sci. Nutr. 1996, $47,5-13$

(37) Kuo, C. C.; Chiang, W.; Liu, G. P.; Chien, Y. L.; Chang, J. Y.; Lee, C. K.; Lo, J. M.; Huang, S. L.; Shih, M. C.; Kuo, Y. H. 2,2'-Diphenyl-1-picrylhydrazyl radical-scavenging active components from adlay (Coix lachryma-jobi L. Var. ma-yuen Stapf) hulls. J. Agric. Food Chem. 2002, 50, 5850-5855.

(38) Nagao, T.; Otsuka, H.; Kohda, H.; Sato, T.; Yamasaki, K. Benzoxazinone from Coix lachryma-jobi var. ma-yuen. Phytochemistry 1985, 24, 2959-2962.

(39) Otsuka, H.; Takeuchi, M.; Inoshiri, S.; Sato, T.; Yamasaki, K. Phenolic compounds from Coix lachryma-jobi var. ma-yuen. Phytochemistry 1989, 28, 883-886. 
(40) Takahashi, M.; Konno, C.; Hikino, H. Isolation and hypoglycemic activity of coixans A, B, and C, glycans of Coix lachryma-jobi L. var. ma-yuen seeds. Planta Med. 1986, 51, 6465.

(41) Tanimura, A. Studies on the antitumor components in the seeds of Coix lachryma-jobi L. var. ma-yuen (Roman.) Stapf. II. The structure of coixenolide. Chem. Pharm. Bull. 1961, 9, 47-53.

(42) Aruoma, O. I. Antioxidant actions of plant foods: use of oxidative DNA damage as a tool for studying antioxidant efficacy. Free Radical Res. 1999, 30, 419-427.

(43) Chan, W. S.; Wen, P. C.; Chiang, H. C. Structure-activity relationship of caffeic acid analogues on xanthine oxidase inhibition. Anticancer Res. 1995, 15, 703-707.

(44) Chen, C. C.; Chen, H. Y.; Shiao, M. S.; Lin, Y. L.; Kuo, Y. H.; Ou, J. C. Inhibition of low-density lipoprotein oxidation by tetrahydrofurofuran lignans from Forsythia suspensa and Magnolia coco. Planta Med. 1999, 65, 709-711.

(45) Conney, A. H.; Lysz, T.; Ferraro, T.; Abidi, T. F.; Manchand, P. S.; Laskin, J. D.; Huang, M. T. Inhibitory effect of curcumin and some related dietary compounds on tumor promotion and arachidonic acid metabolism in mouse skin. Adv. Enzyme Regul. 1991, 31, 385-396.

(46) Fernandez, M. A.; Saenz, M. T.; Garcia, M. D. Anti-inflammatory activity in rats and mice of phenolic acids isolated from Scrophularia frutescens. J. Pharm. Pharmacol. 1998, 50, 11831186.

(47) Laranjinha, J.; Almeida, L.; Madeira, V. Reduction of ferrylmyoglobin by dietary phenolic acid derivatives of cinnamic acid. Free Radical Biol. Med. 1995, 19, 329-337.
(48) Toda, S.; Kumura, M.; Ohnishi, M. Effects of phenolcarboxylic acids on superoxide anion and lipid peroxidation induced by superoxide anion. Planta Med. 1991, 57, 8-10.

(49) Bouic, P. J.; Clark, A.; Lamprecht, J.; Freestone, M.; Pool, E. J.; Liebenberg, R. W.; Kotze, D.; Van Jaarsveld, P. P. The effects of B-sitosterol (BSS) and B-sitosterol glucoside (BSSG) mixture on selected immune parameters of marathon runners: inhibition of post marathon immune suppression and inflammation. Int. J. Sports Med. 1999, 20, 258-262.

(50) Bouic, P. J.; Etsebeth, S.; Liebenberg, R. W.; Albrecht, C. F.; Pegel, K.; Van Jaarsveld, P. P. $\beta$-Sitosterol and $\beta$-sitosterol glucoside stimulate human peripheral blood lymphocyte proliferation: implications for their use as an immunomodulatory vitamin combination. Int. J. Immunopharmacol. 1996, 18, 693700.

(51) Donald, P. R.; Lamprecht, J. H.; Freestone, M.; Albrecht, C. F.; Bouic, P. J.; Kotze, D.; Van Jaarsveld, P. P. A randomised placebo-controlled trial of the efficacy of $\beta$-sitosterol and its glucoside as adjuvants in the treatment of pulmonary tuberculosis. Int. J. Tuberc. Lung Dis. 1997, 1, 518-522.

(52) Gupta, M. B.; Nath, R.; Srivastava, N.; Shanker, K.; Kishor, K.; Bhargava, K. P. Anti-inflammatory and antipyretic activities of $\beta$-sitosterol. Planta Med. 1980, 39, 157-163.

Received for review November 26, 2002. Revised manuscript received March 21, 2003. Accepted March 21, 2003. This research work was supported by the Department of Health, Taiwan, Republic of China, under Grants DOH88-TD-1048 and DOH89-TD-1070.

JF021154W 